Émilien DUBIEZ ${ }^{1,2}$

Cédric Vermeulen ${ }^{1,3}$

Jean Philippe Tonneau 4

Timothée YAmBA YAMBA ${ }^{2}$

Baby MVOLO

Adélaïde LARZILLIÈrE ${ }^{2}$

${ }^{1}$ Cirad

UR B\&SEF

Campus international de Baillarguet

34398 Montpellier Cedex 5

France

${ }^{2}$ Cirad - Projet Makala

57, avenue des Sénégalais

La Gombé

Kinshasa

République démocratique du Congo

${ }^{3} \mathrm{ULg} /$ Gembloux Agro-Bio Tech

Unité GRFMN

Laboratoire de foresterie tropicale

et subtropicale

Passage des Déportés, 2

5030 Gembloux

Belgique

${ }^{4}$ Cirad

Umr TETIS

Campus international de Baillarguet 34398 Montpellier Cedex 5

France

\section{Le paysage comme outil
d'aménagement des terroirs \\ Le paysage comme outil
d’aménagement des terroirs villageois}




\section{RÉSUMÉ}

\section{LE PAYSAGE COMME OUTIL D'AMÉNAGEMENT DES TERROIRS VILLAGEOIS}

La gestion de la ressource bois énergie en périphérie des grands centres urbains est devenue une préoccupation de premier ordre en Afrique centrale. À une demande croissante en bois énergie correspondent des auréoles de déforestation de plus en plus larges. Les communautés villageoises situées dans cette auréole vivent aujourd'hui dans un environnement à ce point dégradé que les écosystèmes forestiers et les biens et services associés y ont presque disparu. La surexploitation de ces espaces nécessite entre autres de repenser l'aménagement des terroirs villageois. Cette étude présente une démarche d'élaboration de plan simple de gestion de terroirs villageois. L'article discute la pertinence d'une approche d'aménagement fondée sur le paysage perçu et vécu par les populations. L'organisation sociale de la communauté, ses pratiques et ses modes de régulation d'accès à la ressource sont des éléments qui structurent le paysage. Ils se traduisent dans une typologie locale de l'espace employée au quotidien. Cette typologie a été mobilisée pour une meilleure appropriation des concepts d'aménagement par les communautés et pour une mise en œuvre plus effective. À contre-courant de la foresterie communautaire développée depuis plusieurs années en Afrique centrale, cette approche est structurée autour d'un processus participatif, progressif et itératif. Elle permet d'amener des groupes endogènes à intégrer leur vision de l'espace dans les prises de décision, à faciliter les débats autour de la question de l'aménagement de leur terroir et à contribuer à la reconstitution d'espaces forestiers dégradés.

Mots-clés : paysage, typologie locale, aménagement, bois énergie, Bas-Congo, République démocratique du Congo.

\section{ABSTRACT}

\section{PERCEPTIONS OF LANDSCAPE IN THE DEVELOPMENT OF VILLAGE LANDS}

The management of fuelwood in periurban areas has become a major concern in Central Africa, where growing demand for fuelwood is rapidly increasing the radius of deforestation around its cities. The village communities within these areas are living in an environment that has become degraded to the point where forest ecosystems and their associated goods and services have almost disappeared. The over-exploitation of these areas demands new thinking on the management of community lands. This paper presents a simple management planning approach for community areas. The article discusses the relevance of a management approach based on landscape as perceived and used by the local populations themselves. The structure of these landscapes has evolved with each community's pattern of social organization, practices and rules of access to resources, all of which translate into the typology of a local landscape in daily use. This typology has been called upon to strengthen community appropriation and implementation of development concepts. In contrast with the community forestry approach that has developed in Central Africa for several years, this approach is built around a participatory, iterative, step-by-step process that allows endogenous groups to bring their own vision of their landscape into decision-making, to facilitate discussions around village land management issues and to contribute to the rehabilitation of degraded forest areas.

Keywords: landscape, local typology, management, fuelwood, Lower Congo, Democratic Republic of Congo.

\section{RESUMEN}

\section{EL PAISAJE COMO HERRAMIENTA DE MANEJO DE TIERRAS COMUNALES}

La gestión de los recursos de leña en la periferia de los grandes centros urbanos se ha convertido en una preocupación de primer orden en África Central. La creciente demanda de leña se refleja en unas "aureolas" de deforestación cada vez mayores. Las comunidades campesinas ubicadas en dichas áreas viven en un entorno tan degradado que los ecosistemas forestales y los bienes y servicios asociados casi han desaparecido. La sobre explotación de estas áreas requiere, entre otras cosas, replantearse el manejo de las tierras comunales. Este estudio presenta una iniciativa de elaboración de un plan simple de ordenación de tierras comunales. En este artículo se examina la pertinencia de un enfoque de manejo basado en el paisaje tal como lo percibe y vive la población. La organización social de la comunidad, sus prácticas y modos de regulación de acceso a los recursos, son elementos que estructuran el paisaje y que se reflejan en una tipología local del espacio empleado cotidianamente. Se ha desplegado esta tipología para permitir una mejor apropiación de los conceptos de manejo por parte de las comunidades y lograr una aplicación más eficaz. Este enfoque, a contracorriente de la silvicultura comunitaria que viene desarrollándose desde hace varios años en África Central, se estructura en torno a un proceso participativo, progresivo e iterativo. Este planteamiento posibilita que los grupos endógenos integren su visión del espacio en las tomas de decisión, facilita los debates relativos a la cuestión del manejo de su territorio y la contribución a la reconstitución de espacios forestales degradados.

Palabras clave: paisaje, tipología local, manejo, leña, Bajo Congo, República Democrática del Congo. 


\section{L'enjeu de la gestion des ressources forestières en périphérie des centres urbains en Rdc}

La République démocratique du Congo (Rdc) compte près de 155,5 millions d'hectares de forêts (EBA'A ATYI, BAYOL, 2009), ce qui en fait le deuxième pays tropical forestier au monde. Ses écosystèmes forestiers, au cœur des enjeux sur le climat, sont des lieux de vie pour des populations rurales dépendant encore fortement des biens et des services fournis par les espaces naturels. Il est aujourd'hui demandé en outre à ces espaces de favoriser le maintien de services écosystémiques tels que la fixation de carbone, la lutte contre l'érosion, tout en continuant à produire des biens dont les populations rurales bénéficient au quotidien. Or l'approvisionnement en bois énergie de la capitale Kinshasa, estimé à 4,8 millions de $\mathrm{m}^{3}$ /an (SCHURE et al., 2011), conduit à la détérioration des massifs forestiers et à la disparition progressive des biens et services disponibles. Le taux de déforestation dans le bassin d'approvisionnement en bois énergie de la ville de Kinshasa a été en effet estimé à $0,7 \%$ par an, supérieur au taux de déforestation moyen à l'échelle du pays (0,25\% par an) (CN REDD RDC, 2011).

Les effets conjugués de cette exploitation pour le bois énergie, de la pression foncière pour l'agriculture, de l'économie de marché et de la déculturation des sociétés ont conduit en outre à des modifications de la gestion traditionnelle des espaces villageois (VERMEULEN et al., 2011). Toutefois, dans certaines zones, comme les districts de la Lukaya et des Cataractes, situés dans la province du Bas-Congo au Sud de la ville de Kinshasa, des espaces forestiers ont été conservés ou sont reconstitués. Ces espaces forestiers issus des modes de gestion traditionnels endogènes marquent le paysage et sont parfois les derniers espaces boisés présents. Ils remplissent différentes fonctions telles que fourniture de différents biens et services (compléments alimentaires, produits de la pharmacopée traditionnelle, bois de chauffe), refuge de biodiversité, fonctions sociales, culturelles, symboliques (PELTIER et al., 2010). Pour conserver ces derniers espaces forestiers, leur aménagement et celui des terroirs qui les hébergent s'avèrent indispensables (DUBIEZ et al., 2012). Se pose dès lors la question de l'approche à adopter. Est-il plus pertinent de choisir une gestion prévisionnelle classique basée sur la quantification de la ressource ou d'opter pour une gestion opérationnelle fondée sur la caractérisation de l'espace perçu et vécu par les communautés locales ? Pour ces forêts gérées par des villageois, il a été testé des plans simples de gestion (Psg) qui ont intégré les représentations locales de l'espace.

Cet article qui veut analyser la pertinence de ce choix se structure autour des questions suivantes : l'aménagement de terroir basé sur une approche paysagère est-il adapté à l'échelle locale (conditions sociales, perception...), et permet-il de programmer une gestion de la ressource forestière par les communautés?

Pour répondre à ces questions, la première partie de cet article traite du concept et de la place du paysage dans l'aménagement des terroirs villageois. La deuxième partie décrit la construction du paysage en lien avec l'organisation sociale. La troisième partie décrit la méthodologie utilisée pour caractériser l'espace vécu des populations et présente un exemple d'aménagement. Enfin, l'analyse de la démarche et les résultats appliqués sur les sites d'étude sont présentés et discutés.

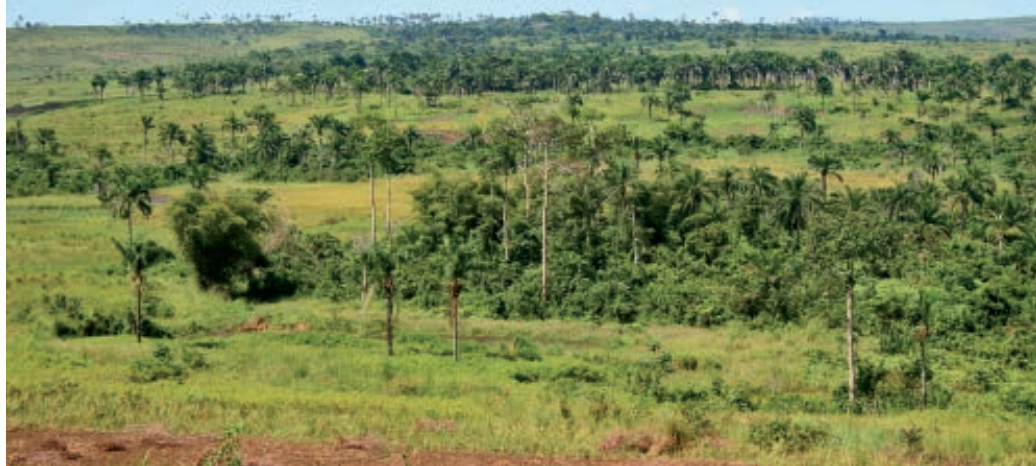

Paysage dégradé du finage du village de Kinduala, province du Bas-Congo, République démocratique du Congo. Photo E. Dubiez, 2010.

\section{Le paysage : porte d'entrée de l'aménagement des terroirs villageois}

En sciences forestières, l'aménagement forestier « classique » passe par la description d'un capital forestier (la ressource disponible), de sa productivité et de sa capacité de renouvellement pour calculer et programmer un prélèvement durable de la ressource. C'est ainsi que sont aménagées autant les forêts tempérées que les concessions forestières et les forêts communautaires d'Afrique centrale (JULVE et al., 2007). De nombreux auteurs ont cependant montré que le développement de la foresterie communautaire a été entravé par cette approche, peu appropriée par les populations (CUNY et al., 2004 ; VERMEULEN et al., 2006 ; EZZINE DE BLAS et al., 2011). L'aménagement forestier classique, basé sur une mise en valeur rationnelle et durable du stock, n'est en outre pas adapté à des écosystèmes fortement anthropisés où l'arbre est rare (VERMEULEN et al., 2011).

La gestion de terroir est souvent avancée comme une alternative. C'est «l'association des acteurs locaux à la gestion proprement dite du terroir. Elle n'implique pas a priori de dispositif institutionnel particulier. Le concept de gestion de terroir repose essentiellement sur la mise en place de dispositifs permettant d'associer les populations locales à la planification des activités collectives » (MERAL in BALLET, 2007).

Mais comment traduire cette volonté d'associer les acteurs locaux? L'ambition est de proposer, en utilisant la notion de paysage, une approche de l'aménagement proche des pratiques endogènes de gestion. L'approche paysagère, caractérisant la relation entre l'action anthropique et son impact spatial, est privilégiée en considérant que le paysage résulte des modes d'occupation des sols et des pratiques d'exploitation (LAQUES, 2003).

L'évolution du paysage dépend à la fois des processus naturels et des aménagements humains, des perceptions et des idéologies. "Le concept de paysage est un révélateur des dynamiques passées et en cours dans un territoire et il peut être un moyen d'action sur ce territoire » (GAUTIER, 1995). Le paysage permet d'intégrer une dimension physique, réelle 
(pouvant être mesurée : sols, ressources, biomasses...), une dimension « représentation » que s'en font les populations (symbolique, culturelle, productive...) et une dimension « action ». La « représentation » (BRUNET et al., 1993) est définie comme étant «à la fois un processus, qui permet de faire connaître, de rendre compréhensible un phénomène, une idée, un objet (...) et le résultat de ce processus : une

Position des provinces de Kinshasa et du Bas-Congo en RDC

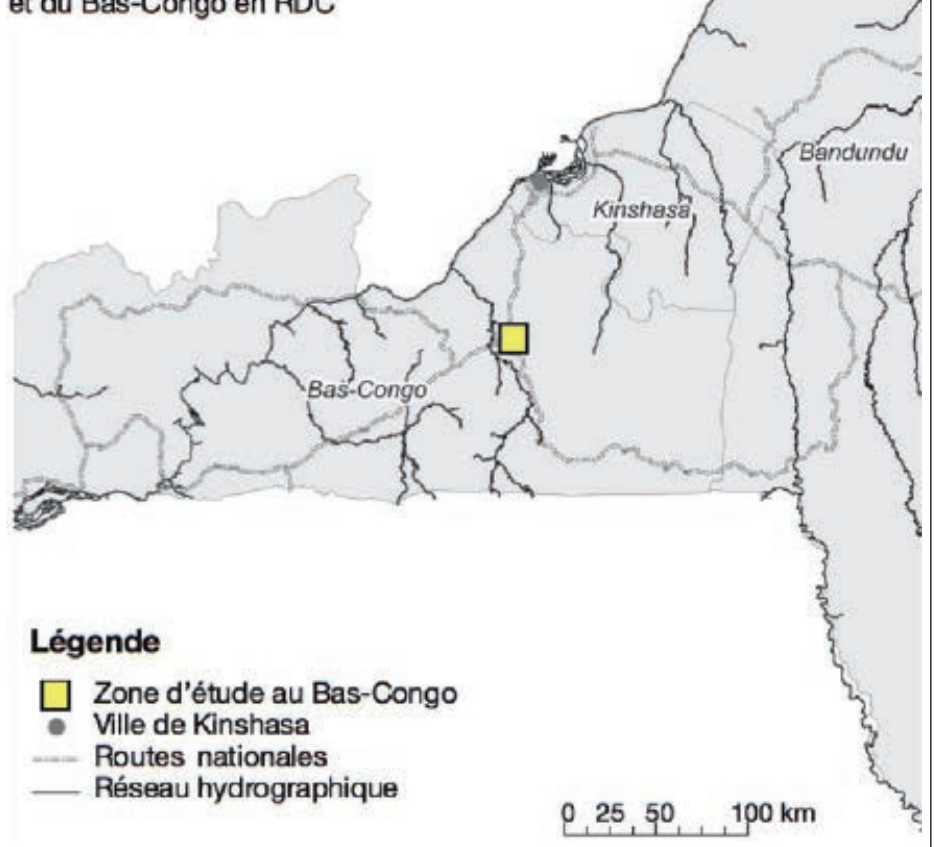

Figure 1.

Situation géographique de la zone d'étude du projet Makala dans la province du Bas-Congo, République démocratique du Congo (VERMEULEN et al., 2011). image, une carte, un diagramme, un tableau, un modèle... ".

L'hypothèse est qu'un aménagement qui s'appuiera sur les représentations des populations locales, sur leur mise en perspective en fonction des nouveaux enjeux des services environnementaux, permettra une meilleure prise en compte des besoins et des aspirations villageoises tout en assurant des décisions plus adéquates et mieux partagées.

\section{L'organisation sociale endogène à l'origine de la construction du paysage actuel}

Dans les villages Batandu du Bas-Congo, l'organisation sociale est structurée autour des lignées. Elles sont composées d'un ensemble de personnes se reconnaissant un ancêtre commun identifiable. Ces lignées sont regroupées en une communauté de résidence, le village administratif (VERMEULEN et al., 2011). Le groupe ethnolinguistique Ntandu possède un système de filiation de type matriarcal. Les chefs de lignée sont responsables de la gestion de leur terroir (distribution de terre, respect des règles d'usage...). Par le passé, ces lignées étaient mobiles dans l'espace. Cette mobilité avait pour origine des conflits familiaux ou une volonté de trouver de meilleures conditions de vie. Les migrations pouvaient s'effectuer sur des espaces réduits. C'est le cas par exemple du village de Kinduala qui possède un finage d'environ 850 ha pour une population actuelle de 435 personnes (DUBIEZ et al., 2009). Ce village est composé de trois lignées.

Le déplacement de ces lignées par le passé est à l'origine du découpage actuel du finage villageois en plusieurs terroirs sous la gestion chacun d'un lignage (figure 2). Dans ce finage se trouvent 16 sites d'anciens villages (Voka en lingala) : 11 sont issus des migrations des différentes lignées au sein du finage et 5 ont été hérités d'un village voisin par relation matrilinéaire.

Du fait de la pression agricole ou de la production de charbon de bois (makala en lingala), une partie de ces anciens villages a aujourd'hui été déboisée. Les sites déboisés ne sont plus composés que de palmiers à huile (Elaeis guineensis), associés à

Figure 2.

Mouvements migratoires récents dans le finage du village de Kinduala à l'origine de l'implantation des anciens villages, aujourd'hui derniers espaces forestiers de la zone d'étude, province du BasCongo, République démocratique du Congo (VERMEULEN et al., 2011). 


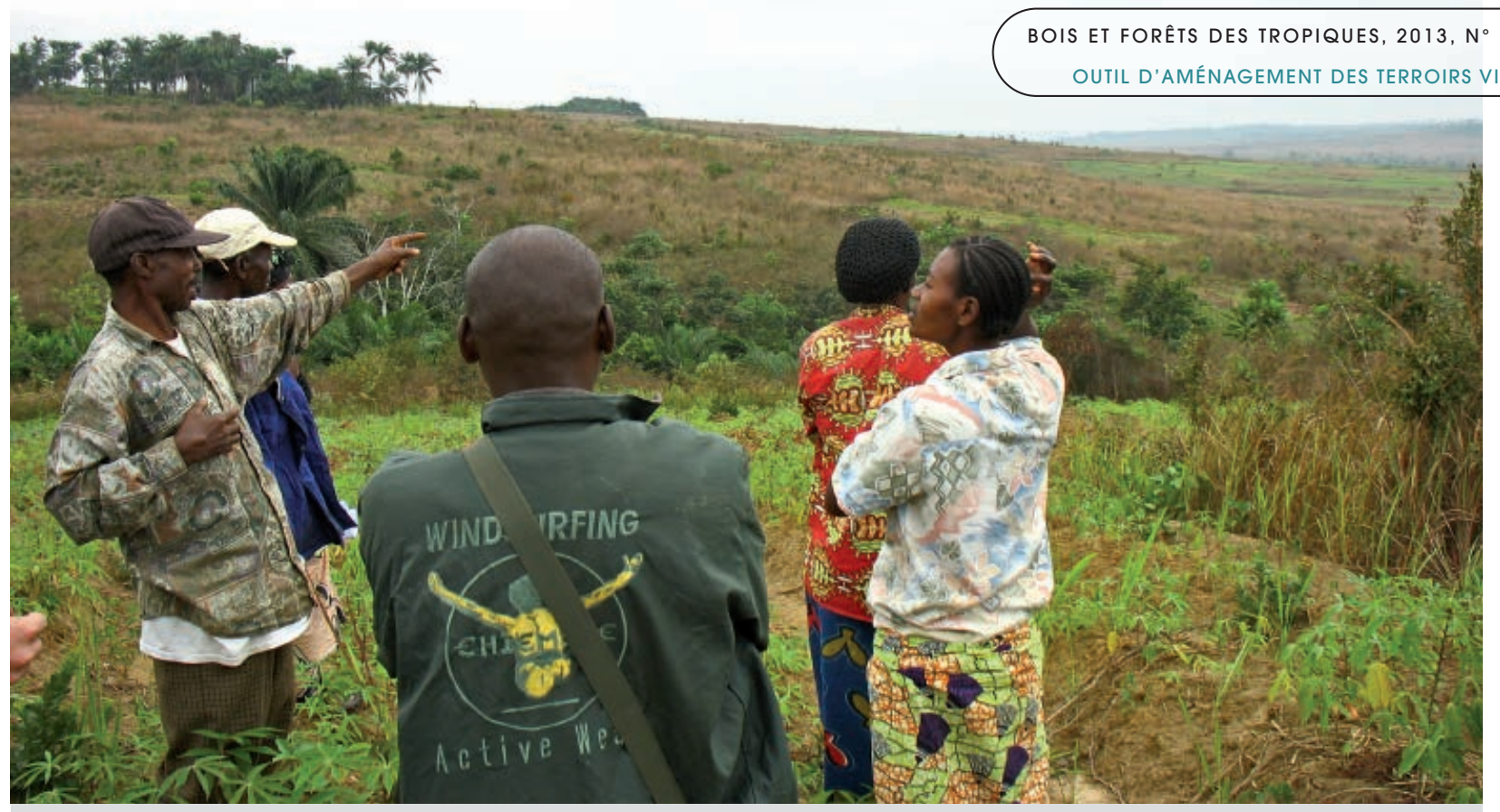

Visite de terroir, avec un groupe de travail, pour caractériser les différentes unités paysagères perçues sur le terroir. Photo A. Larzillière, 2011.

des cultures (Arachis hypogaea, Manihot esculenta...). Nous en comptons actuellement 11 de ce type dans le finage de Kinduala. D'autres sites, à l'inverse, présentent encore un réel caractère forestier. Ils sont au nombre de cinq dans le finage aux superficies comprises entre 0,2 ha et 1 ha. Ils sont formés en partie d'essences forestières (Pentaclethra eetveldeana, Pseudospondias microcarpa, Caetocarpus africana, Dichostemma glaucescens...) et de fruitiers plantés au cours de l'occupation passée des villages.

Un autre élément du paysage est également issu des modes de gestion traditionnels de l'espace par les populations Batandu : il s'agit du Nkunku. Le Nkunku est un espace donné que la lignée décide de mettre en défens avec deux objectifs principaux, la reconstitution de la fertilité des sols et la fourniture de bois (bois énergie et/ou bois de construction) (PELTIER et al., 2010).

L'exemple des Mavoka et des Nkunku, structurant le paysage des terroirs villageois des districts de la Lukaya et des Cataractes, montre donc que les Batandu façonnent depuis longtemps, profondément, le paysage.

Il apparaît donc pertinent de s'appuyer sur la représentation locale de l'espace et sur les typologies employées par les populations Batandu comme outil d'aide à l'aménagement des terroirs villageois. Cette typologie évite l'emploi d'un vocabulaire technocratique étranger au quotidien villageois. À la parcelle de l'aménagiste se substitue le toponyme local. Le lignage est considéré comme l'unité sociale pour définir l'aménagement. De fait, l'unité spatiale à aménager est l'espace contrôlé par la lignée, le terroir. Le terroir (KARSENTY, MARIE, 1997) étant considéré comme l'ensemble des terres soumises au cycle cultural incluant les jachères et recrûs forestiers, et comme étant une portion du finage où les logiques d'occupation du sol sont dominantes. Le groupe endogène choisi, le lignage repose sur sa légitimité sociale et non sur un caractère administratif ou associatif imposé de l'extérieur.

\section{Caractérisation de l'espace sous gestion}

La figure 3 présente les cinq étapes méthodologiques préalables à la définition des mesures d'aménagement. Elles permettent de délimiter l'espace géré par le lignage, de recueillir la typologie locale, de diviser l'espace en unités paysagères, de valider la perception avec la communauté et d'identifier les lieux-dits au sein du terroir. Ces étapes sont élaborées par des représentants de la lignée, regroupés en une assemblée endogène légitime. Les membres sont choisis par la communauté pour représenter la diversité sociale (femmes, hommes, jeunes, vieux...).

\section{Élaboration d'une carte schématique de l'espace sous gestion du lignage}

Cette étape permet de caractériser l'espace, le terroir, où s'exercent les droits traditionnels d'accès et d'usage du lignage. L'espace sous gestion est d'abord représenté et débattu collectivement à l'aide d'une maquette interactive (LARZILLIÈRE et al., présent dans ce numéro, 2013), avant d'être représenté schématiquement sur papier en respectant les proportions des différents éléments décrits (cartes à dire d'acteurs). Les éléments représentés sur ce schéma sont les routes, les rivières, les sentiers, les sources d'eau... c'est-à-dire tous les éléments remarquables et repérables de l'espace qui permettent de se situer aisément.

\section{Identification de la typologie locale}

Les membres de l'assemblée endogène décrivent de manière concertée les différents éléments du paysage perçus et les nomment en langue Kitandu. Cette typologie locale est ensuite localisée collectivement sur la carte schématique au cours de plusieurs sorties de terrain. 


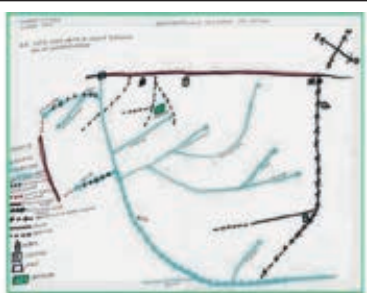

Schèma de l'espace sous restion
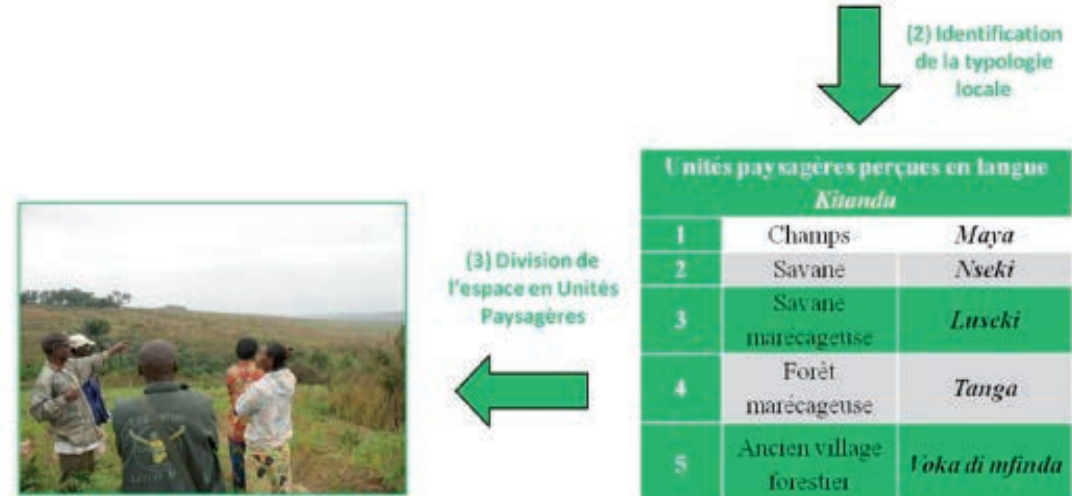

Visite de terroir avecun sroupe de travail
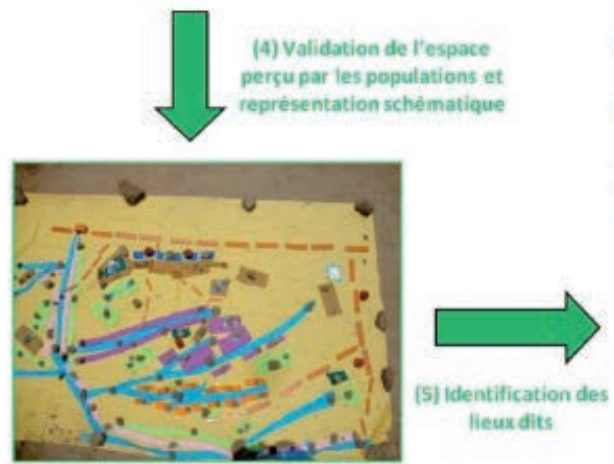

Maguette interactive grêsentant les Unité Paysagères décrites gar la communauté
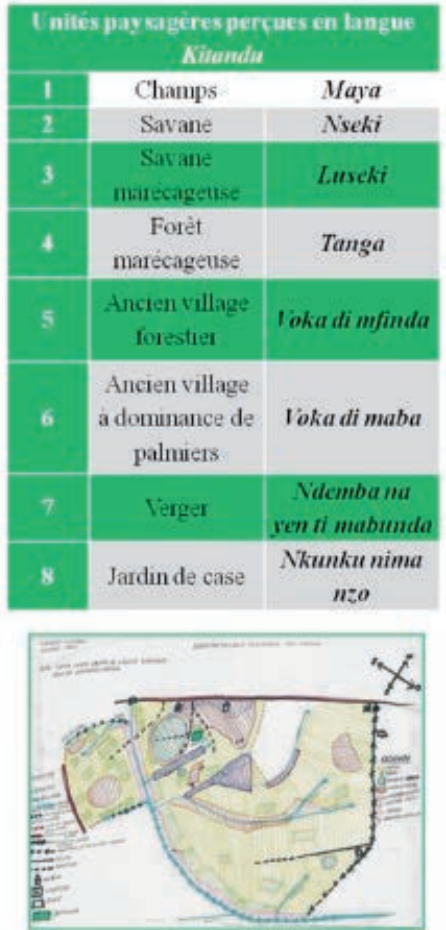

Schéma des Unités Paysagères assorti des lleux dits

Figure 3.

Méthodologie pour la détermination de la typologie locale et la caractérisation des unités paysagères par les populations locales.

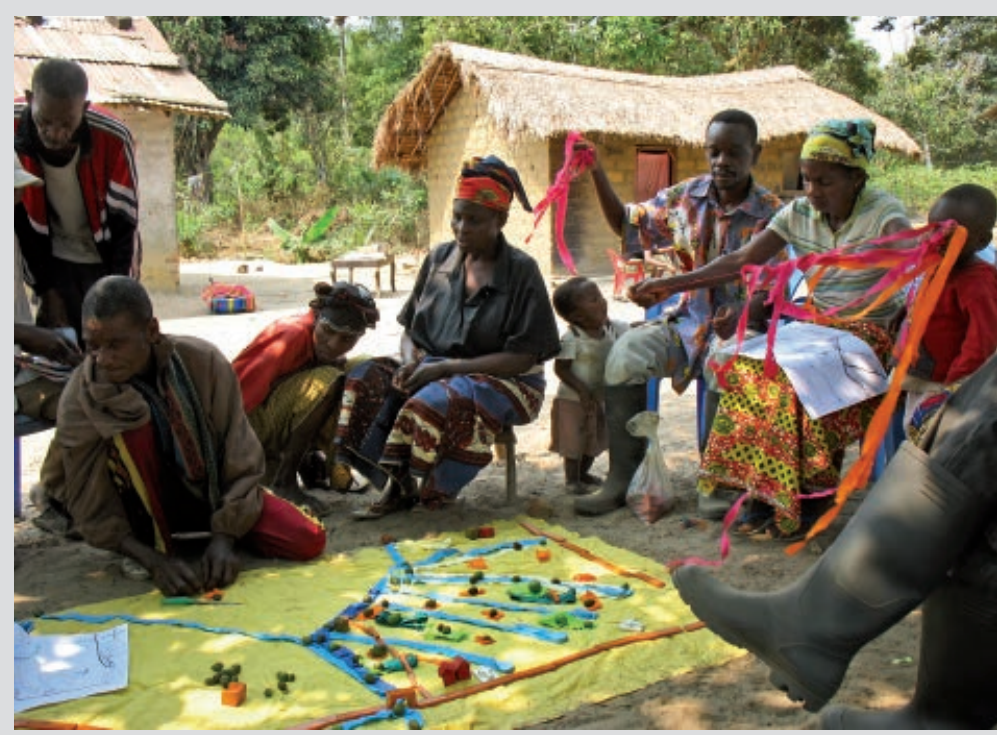

Représentation des unités paysagères, définies lors de la sortie de terrain, à l'aide de la maquette interactive. Photo A. Larzillière, 2011.

\section{Division de l'espace en unités paysagères}

Durant ces mêmes sorties, l'espace est divisé en unités paysagères fondées sur cette typologie locale.

\section{Validation de l'espace perçu par les populations et représentation schématique}

Les unités paysagères identifiées au cours de la sortie de terrain sont ensuite validées à l'aide de la maquette interactive. Cette étape permet de représenter en trois dimensions les différents espaces perçus et décrits au sein du terroir sous gestion et de les soumettre au débat collectif.

\section{Identification des lieux-dits}

Chaque unité paysagère représentée est enfin dénommée selon les toponymes locaux afin de découper le terroir en espaces de gestion distincts les uns des autres et nommés selon les lieux-dits d'usage local.

Ces différentes étapes permettent de caractériser les représentations qu'ont les communautés de leur espace, de la manière dont elles l'occupent et elles le vivent. Comme le rappelle PIVETEAU (1995) : « Le territoire est à la fois le cadre spatial dans lequel s'inscrivent les pratiques et les représentations spatiales d'une société et le produit de ces représentations. " Ce travail permet donc d'obtenir une carte des unités paysagères perçues et vécues, utilisée comme outil d'aide à la décision pour la définition des futures mesures d'aménagement au sein du terroir villageois.

Si la typologie locale du paysage (figure 3) décrite par les membres du groupe de travail est analysée, il est constaté que la majorité de ces éléments ont une origine anthropique : les jardins de case, les vergers, les deux types d'anciens villages (forestiers et à dominance de palmiers) ainsi que les champs. Dans cette typologie, la jachère forestière n'apparaît pas. Cela s'explique par la pression foncière importante dans la zone, qui se traduit par des rotations culturales courtes empêchant toute reconstitution de la forêt. Les seuls espaces forestiers sont des espaces issus de la gestion traditionnelle de l'espace par les Batandu. Il s'agit des vergers (Ndimba na yen ti mabunda), des jardins de case (Nkunku nima nzo) et des anciens villages forestiers (Voka di mfinda). Les forêts marécageuses (Tanga) sont maintenues en partie en raison de leur faible accessibilité. 


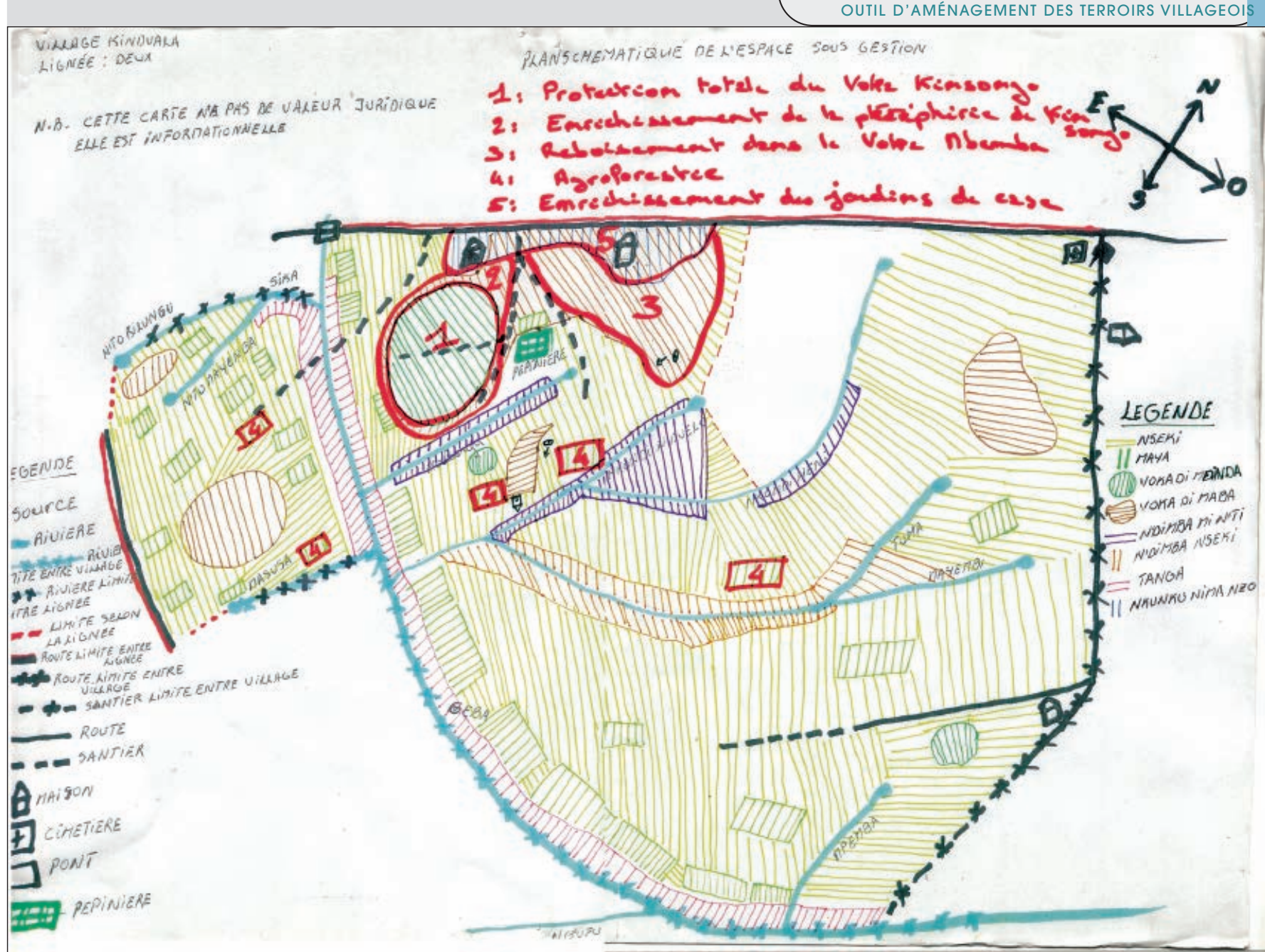

Figure 4.

Modalités de gestion définies par la deuxième lignée du village de Kinduala (province du Bas-Congo, République démocratique du Congo, 2012). Protection totale de l'ancien village forestier de Kinsongo. L'objectif est de conserver cet ancien village forestier, lieu de sépulture des ancêtres et source de différents biens et services. Enrichissement en essences locales de la périphérie de l'ancien village forestier de Kinsongo avec des plants produits en pépinière par la lignée. Dans ce cas, les villageois ont décidé que l'activité de plantation serait collective. Reboisement de l'ancien village à dominance de palmiers, Mbemba. L'objectif est de reconstituer un espace forestier à l'aide d'essences locales choisies par le lignage en fonction de leurs intérêts. Les plants sont installés, collectivement, dans les champs existants pour faciliter l'entretien de ces derniers. Boisement d'Acacia auriculiformis en champ. Les plants sont produits individuellement par les membres intéressés de la lignée. Les objectifs sont de reconstituer la fertilité des sols, de produire du bois à court terme et de favoriser la protection des espaces forestiers relictuels en proposant une source de bois alternative. Enrichissement des jardins de case en essences fruitières. Cette activité est individuelle.

\section{L’aménagement : exemple de la deuxième lignée du village de Kinduala}

La caractérisation de l'espace terminée, la phase d'aménagement peut débuter. Chaque élément du paysage décrit sert de passerelle à la définition des futures modalités de gestion au sein du terroir. Les modalités de gestion sont elles aussi représentées sur le schéma des unités paysagères (figure 4) et débattues collectivement par l'ensemble de la lignée. Pour chaque mesure de gestion retenue, des règles et des interdits sont ensuite définis par les membres du groupe de travail et partagés avec l'ensemble de la communauté. Ils ont pour objectifs de pérenniser les mesures retenues en définissant un cadre collectif de gestion. La répartition des bénéfices est également discutée préalablement pour éviter tout conflit ultérieur au moment de la valorisation de ces espaces.

Dans le cas de la deuxième lignée du village de Kinduala, cinq modalités de gestion ont été retenues par les villageois pour l'aménagement de leur terroir et pour la reconstitution d'espaces forestiers.

Actuellement, plus de 2300 plants d'essences locales (Millettia laurentii, Pentaclethra macrophylla, Pentaclethra eetveldeana, Pseudospondias microcarpa, Maesopsis eminii...) ont été introduits dans le terroir de la deuxième lignée du village de Kinduala et 3000 plants d'Acacia auriculiformis dans les champs. 


\section{Discussion}

\section{Quantification ou appropriation ?}

La question illustrée est opérationnelle : est-il plus pertinent d'opter pour une gestion prévisionnelle classique basée sur la connaissance de la ressource ou de choisir une approche opérationnelle fondée sur la perception de l'espace par les populations et sur l'utilisation de la typologie locale pour l'aménagement des terroirs villageois ? Le tableau I présente les intérêts et les inconvénients de ces deux approches pour l'élaboration d'un modèle d'aménagement de la ressource forestière d'un terroir.

En résumé, l'approche spatiale fondée sur une vision quantifiée rigoureuse a le mérite de la rationalité mais peu de villageois la comprennent, donc se l'approprient. Elle est supposée être plus efficace mais en pratique elle est souvent peu ou pas appliquée. Chaque société humaine possède des modèles de gestion plus ou moins élaborés et plus ou moins efficaces face aux différents enjeux. L'enjeu n'est pas de créer ex nihilo une gestion de la ressource arborée mais de faire évoluer les pratiques pour prendre en compte de nouvelles exigences. En ce sens, l'approche par le paysage vécu se révèle avantageuse.

\section{Vers une réappropriation collective de la gestion}

L'élaboration du plan simple de gestion a obligé chaque villageois à expliciter son espace perçu et vécu. L'exercice s'est révélé particulièrement aisé pour la population. Les échanges et les débats s'organisent autour d'éléments visibles et connus des usagers de l'espace. La population décrit le paysage qu'elle voit avec la typologie qu'elle utilise au quotidien. Les visites de terrain, organisées en groupe, permettent également une réappropriation collective de l'espace, une mutualisation et une réactualisation des savoirs. Aborder l'analyse du paysage permet en outre aux villageois de prendre conscience de l'évolution temporelle de ce dernier et de comprendre l'impact de leurs pratiques sur le milieu.

L'autre intérêt de cette démarche est le faible coût de sa mise en œuvre. Il n'est pas nécessaire de disposer de matériel onéreux et de technologies avancées : seuls l'observation et les échanges sont à l'origine de la construction de l'aménagement. La mise en place du processus participatif avec une communauté prend toutefois du temps et nécessite une proximité importante entre les acteurs du projet et la communauté ciblée.

Tableau I.

Choisir une gestion opérationnelle ou prévisionnelle. Présentation des avantages et inconvénients d'une programmation opérationnelle reposant sur l'analyse paysagère et d'une programmation prévisionnelle basée sur des inventaires.

Avantages

\author{
Gestion de la ressource forestière d'un terroir villageois \\ Analyse du paysage \\ Gestion opérationnelle \\ Inventaires forestiers \\ Gestion prévisionnelle
}

Connaissance de la ressource

Repose sur la typologie locale de l'espace (facilite l'appropriation)

Réappropriation collective de l'espace

Actualisation de la typologie locale de l'espace

Facile à mettre en œuvre

Facilement compréhensible

Coût de mise en œuvre faible

Modification des représentations et perceptions des populations par le discours du projet

Pas de quantification de la ressource à gérer
Coût important

Temps de mise en œuvre long

Difficilement compréhensible par les populations

Non adapté à des espaces fortement dégradés 


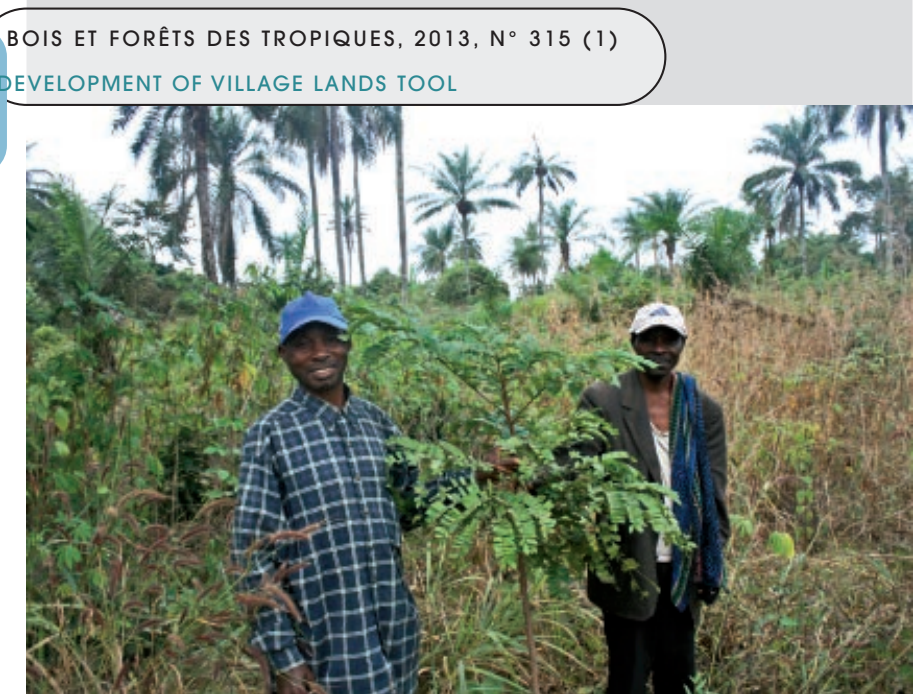

Photo 7.

Plant de Pentaclethra macrophylla dans le terroir de la deuxième lignée du village de Kinduala. Photo E. Dubiez, 2012.

\section{Références bibliographiques}

BALLET J., 2007. La gestion en commun des ressources naturelles : une perspective critique. Développement durable et territoires. Varia, mis en ligne le 29 août 2007. developpementdurable.revues.org

BRUNET R., FERRAS R., THÉRY H., 1992 [1993]. Les mots de la géographie : dictionnaire critique. Montpellier/Paris, France, RECLUS/La Documentation française, coll. Dynamique du territoire, $3^{e}$ édition.

COORDINATION NATIONALE REDD RÉPUBLIQUE DÉMOCRATIQUE DU CONGO, 2011. Plan d'investissement pour le Programme d'investissement pour la forêt. Kinshasa, Rdc, Ministère de l'Environnement, Conservation de la Nature et Tourisme, $76 \mathrm{p}$.

CUNY P., ABE'ELE P., NGUENANG G. M., DJEUKAM R., EBOULE S., EYENE E., 2004. États des lieux de la foresterie communautaire au Cameroun. Yaoundé, Cameroun, Ministère de l'Environnement et des Forêts, $149 \mathrm{p}$.

DUBIEZ E., VERMEULEN C., PELTIER R., INGRAM V., SCHURE J., MARIEN J.-N., 2012. Managing forest resources to secure wood energy supply for urban centers: the case of Kinshasa, Democratic Republic of Congo. Nature \& Faune, 26 (2): 5256. www.fao.org

DUBIEZ E., VERMEULEN C., PROCES P., YAMBA YAMBA T., 2009. Recensement, histoire, occupation spatiale et secteur associatif du village de Kinduala. Projet UE-Makala. CiradGembloux Agro-Bio Tech, 54 p.

EBA'A ATYI R., BAYOL N., 2009. Les forêts de la République Démocratique du Congo en 2008. In : De Wasseige C. et al. (éds). Les forêts du Bassin du Congo - État des Forêts 2008. Luxembourg, Office des publications de l'Union européenne, 49-59.

EZZINE DE BLAS D., RUIZ PEREZ E., VERMEULEN C., 2011. Management conflicts in Cameroonian community forests. Ecology and Society, 16 (1): 8. URL: www.ecologyandsociety.org
GAUTIER D., 1995. La délimitation des paysages : exemple de la Vallée Française en Cévennes. Mappemonde, 3 : 35-39.

JULVE C., VANDENHAUTE M., VERMEULEN C., CASTADOT B., EKODEK H., DELVINGT W., 2007. Séduisante théorie, douloureuse pratique : la foresterie communautaire camerounaise en butte à sa propre législation. Parcs et Réserves, 62 (2) : 18-24. KARSENTY A., MARIE J., 1997. Les tentatives de mise en ordre de l'espace forestier en Afrique Centrale. In : Dynamiques sociales et environnement : pour un dialogue entre chercheurs, opérateurs et bailleurs de fonds. Gret-CnrsOrstom-Banque mondiale.

LAQUES A.-E., 2003. Paysages et modèles paysagers : des indicateurs géographiques pour l'analyse des dynamiques spatio-temporelles d'un front pionnier - le cas de São Félix do Xingu (Brésil, État du Pará). In : Maby J. (dir.). Objets et indicateurs géographiques. Avignon, France, Éditions des Actes d'Avignon n 5, Université d'Avignon, 109-120.

LARZILLIÈRE A., VERMEULEN C., DUBIEZ E., YAMBA YAMBA T., DIOWO S., MUMBERE G., 2013. La maquette interactive, un outil novateur de participation. Bois et Forêts des Tropiques, 315, $10 \mathrm{p}$.

PELTIER R., NSIMUNDELE KONDO L., DIANSAMBU MAKANUA I., PROCES P., DUBIEZ E., MARIEN J.-N., VERMEULEN C., 2010. Conserver ou manger la forêt ? Le paradoxe des paysans en périphérie de Kinshasa, RDC. Aires protégées traditionnelles du Bas-Congo. Le Flamboyant, 66-67 : 33-37.

PIVETEAU J.-L., 1995. Le territoire est-il un lieu de mémoire. L’Espace Géographique, 24 (2) : 113-123.

SCHURE J., INGRAM V., MARIEN J.-N., NASI R., DUBIEZ E., 2011. Woodfuel for urban centres in the Democratic Republic of Congo. The number one energy and forest product returns to the policy agenda. Brief of CIFOR, 7, $2 \mathrm{p}$.

VERMEULEN C., DUBIEZ E., PROCES P., DIOWO MUKUMARY S., YAMBA YAMBA T., MUTAMBWE S., PELTIER R., MARIEN J.N., DOUCET J.-L., 2011. Enjeux fonciers, exploitation des ressources naturelles et Forêts des Communautés Locales en périphérie de Kinshasa, République Démocratique du Congo. Biotechnologie, Agronomie, Société et Environnement, 15 (4) : 535-544.

VERMEULEN C., VANDENHAUTE M., DETHIER M., EKODECK H., NGUENANG G. M., DELVINGT W., 2006. De Kompia à Djolempoum : sur les sentiers tortueux de l'aménagement et de l'exploitation des forêts communautaires au Cameroun. VertigO - La revue électronique en sciences de l'environnement, 17 (1), 8 p. 